\title{
A study on VM-based Terminal Mobility Management
}

\author{
KangWoon Hong, Il-Koo Jung \\ Electronics and Telecommunications Research Institute \\ gwhong@etri.re.kr, ilkoo@etri.re.kr
}

\begin{abstract}
We propose Virtual Machine (VM)-based terminal mobility management. The mechanism requires enhanced mobility control procedures and data transmission procedure comparing with the existing mobility management like Access Independent Mobile Service (AIMS). The procedures newly introduce location notification message from Mobility Information and Control Server (MICS) to VM. The proposed mechanism consequently mitigates a bad effect and improves bottleneck caused by distributed mobility management.
\end{abstract}

Keywords - Virtual machine, location registration, data transmission

\section{INTRODUCTION}

Recently, subscribers are using services utilizing various kinds of virtualization technology. Among different virtualization technologies, virtual Desktop Interface service as a typical one is fascinating IT industry in terms of economic feasibility, safety, device/network diversity, etc. For example, subscribers access Internet via WiFi networks. If getting out of WiFi coverage, WiBro and HSPA access networks are available. Also, the requirement to retain connection will increase as people more frequently utilize smart devices to connect to Internet. Here, in order to efficiently deal with the above user environment, the VMbased terminal mobility management mechanism is critical.

By using the VM-based terminal mobility management technology, we can mitigate some problems in the existing terminal mobility management technologies. Firstly, it is expected for the necessity of cloud-based smart devices to be increased in perspectives of the device cost, the ease of development and so on as subscribers are generally using smart devices like a smartphone and utilization of cloud technology is gradually increased. Secondly, the terminal mobility management technologies can decrease handover latency time or solve the issue for application session retainment depending on whether HoA of terminal is changed after vertical handover. The VM-based terminal mobility management technology retains applications' session without the data loss due to handover latency time. Finally, a micro cloud platform including computing, networking and storage has an opportunity to decrease the latency time critical in VDI services.

In this paper, we introduce Access Independent Mobile Service (AIMS), the terminal mobility management technology among heterogeneous access networks, propose the enhancement to apply the VM-based terminal mobility management to the AIMS and suggest the conclusion.

\section{AIMS SYSTEM}

The technological features are the following.

\section{A. Purpose of AIMS system}

The purpose of AIMS system is to support seamless mobility for a mobile node among heterogeneous access networks in the $\mathrm{BcN}$ environments, which is based on an allIP core network. To accomplish that purpose, features in a technology and structure standpoint are as follows.

- Supports for two separated transport plane: data transport plane and control transport plane

- Support for the data delivery through the optimal route and a distribution scheme of a tunneling overhead

- Support for a fast delivery mechanism for mobility control signals in an core network

- Support for the fast location registration and handover control through the L2/L3 interworking for handover control optimization

- Support for the network selection control based on user preference and network policy

- Supports for the separation of functions of location management and handover control

- Supports for an hierarchical scheme for a mobility control

- Supports for L2/L3 integrated interface supporting various access technologies

\section{B. Functions of AIMS}

The AIMS system is constructed with four functional entities: MICS, Handover Control Agent (HCA), PoA (Point of Attachment), and MN.

- Mobility Information Control Server

The MICS is typically located in the core network and manages the binding information of a MN's L2 identifiers (IDs), HoA and CoA. When a MN performs a handover between different ANs, MICS updates the binding information and controls the IP-in-IP tunnels among HCAs to adjust the data delivery path to the MN's new location. The MICS periodically collects the status information of each AN and then supports a MN's network selection procedure based on the information when the $\mathrm{MN}$ is 
located in an overlapped area of different ANs. The MIH Information service is employed to gather the information and to recommend a target PoA of the MN's handover.

\section{- Handover Control Agent}

The HCA is located at a gateway router connecting the core network and each AN. When it detects a MN's L3 handover, a HCA (at the target AN of handover) allocates the MN's CoA and informs MICS of the MN's CoA and L2 ID. In addition, the HCA performs a location query procedure with MICS to deliver packets to a CN. After the location query procedure, the HCA knows the CN's CoA and establishes an IP tunnel with the $\mathrm{HCA}$ at $\mathrm{CN}$-side.

\section{- Point of Attachment}

After MN's association with a PoA, the PoA immediately provides an upper HCA with a trigger message, which enables the HCA to detect the MN's handover situation. Another function of $\mathrm{PoA}$ is to support the Router Advertisement (RA) caching and proxing operation. After a MN performs a L3 handover, it should change its default gateway to transmit packets in a new routing domain. This change is generally initiated by a RA message periodically broadcasted by access routers. In the AIMS system, a PoA caches the RA message and delivers it immediately after a $\mathrm{MN}$ attaches to the PoA. This can further save a latency required for L3 handover control.

\section{- Mobile Node}

The AIMS architecture requires little functional update to a MN to control L3 mobility since it is designed as a network-based MM scheme. However, to perform the network selection procedure based on user preference and service quality, some functions need to attached to a MN to detect the current link status and search attachable PoAs. For L2 handover control between different ANs, a MN has to manipulate the actions of its various wireless interfaces. Those functions can be implemented based on L2/L3 interactions using the MIH services.

\section{Information flow of AIMS}

To support mobility among heterogeneous access networks, the initial location registration procedure and the location update procedure of handover control are presented in this section. The location management procedure of AIMS system is based on the network-initiated scheme, in which a target PoA initiates a layer 3 handover procedure. In this procedure, network elements use MN's layer 2 (MAC) ID as the key ID to distinguish between MNs.

The location management procedure of AIMS system consists of the procedure of allocating HoA(home address) to a $\mathrm{MN}$ and registering MN's initial location and binding information to MICS, the procedure of selecting a PoA of access network based on the users' preference and a network policy and performing layer 2 handover to target access network, and the procedure of registering new location information to MICS for an handover management among heterogeneous access networks.

\section{Initial registration procedure}

Initial registration procedure for a $\mathrm{MN}$ is initiated by a layer 2 (L2) connection procedure according to an access technology. The AIMS system focus on the layer3-based mobility control technology and the scheme utilizing L2 state information, while a L2 connection scheme and handover control procedure is not included in the AIMS system. To deliver L2 information of many different types of access networks to network layer (L3) as a unified format and support appropriate L2 control for an L3 handover, the L2.5 standard technology such as IEEE 802.21 MIH is applied to AIMS system. Fig. 1 shows the initial location registration procedure of a MN in a AIMS system.

- The procedure is initiated by a MN when it requests a PoA to establish a wireless link (L2 association request in Fig. 1).

- Then the PoA performs an authentication process with an Authentication, Authorization and Accounting (AAA) server to check whether a user (or $\mathrm{MN}$ ) is permitted to access the network. If the AAA server does not know all the L2 IDs of the MN, the information can be updated through the authentication process.

- When a wireless link has been successfully established, the PoA's MIHF will immediately generate an event trigger, called MIH_Link_Up, to notify the MN's attachment.

- The PoA extracts the MN's L2 ID from the MIH_Link_Up trigger and then sends a Location Report message containing the L2 ID to the upper HCA to initiate the network-based $\mathrm{L} 3$ registration procedure.

- Each PoA stores a copy of RA message which is periodically delivered from the gateway router (i.e., HCA). When a trigger indicating a MN's attachment is generated, a PoA immediately sends the cached RA message to the $\mathrm{MN}$. This enables a MN to know its default gateway router with little latency after initial attachment or L2 handover.

- When a HCA receives a Location Report message from a PoA, it searches its own LBT and checks whether the MN's L2 ID is already recorded in the LBT. If an entry containing the MN's L2 ID exists, the HCA determines that the $\mathrm{MN}$ has performed a L2 handover between two PoAs in the same HCA domain. Otherwise, the HCA knows that the $\mathrm{MN}$ has just attached to the network (initial registration case) or has moved from another HCA domain (L3 handover case). Then the HCA creates a new binding entry in its own LBT and records the MN's L2 ID in the binding entry.

- Subsequently, the HCA sends a Location Registration message to inform MICS of the MN's initial attachment or location change. This message contains the MN's CoA (i.e., the IP address of HCA).

- When the MICS receives a Location Registration message, it checks if a binding entry for the $\mathrm{MN}$ already exists in its GBT by searching the MN's L2 ID contained in the message. When the MN just attaches to the network, it is obvious that the MICS has no GBT entry concerned to the MN. Then the MICS creates a new binding entry in its 
GBT and records the MN's CoA (HCA1's IP address) and all L2 IDs.

- A list of a MN's L2 IDs may be obtained from a subscriber management server (represented as AAA server) managing the profiles of subscribers and their terminal devices.

- After that, the MICS sends a Location Registration ACK message to HCA, which a HoA value inside the message is configured to NULL. This enables the HCA to perceive the MN's initial registration situation.

- On receiving the acknowledge message, HCA allocates a HoA for the MN and records it into the LBT. The HoA can be allocated from the HCA's IP address pool or through Dynamic Host Configuration Protocol (DHCP) transaction.

- Finally, the HCA sends a HoA Update messages to the MICS and MN to notify the HoA, respectively. Subsequently, the MICS writes the HoA into a GBT entry concerned with the $\mathrm{MN}$ and then replies to the HCA with a HoA Update ACK message. At the same time, the MN configures its IP address with the HoA and also responses to HCA with a HoA Update ACK message. This completes the initial registration procedure of the $\mathrm{MN}$.

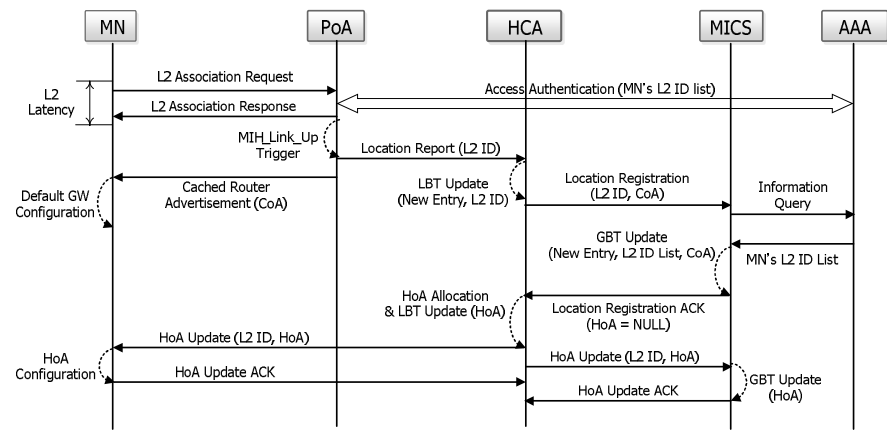

Figure 1. Information Flow in Initial Location Registration

\section{Handover control procedure}

The handover control for a $\mathrm{MN}$ is divided into two cases: handover control within a single access network and handover control among heterogeneous access networks. Handover control within a single access network is limited to L2 handover control according to access network technologies. If there are multiple IP domains in a single access network, it is necessary to consider L3 handover control for a MN and L3 handover control for this access network needs to consider an hierarchical network architecture to manage multiple IP domains. In this documentation, AIMS system focuses on L3 handover control among heterogeneous access networks so that it is considered an access network consists of only one IP domain and is managed by one HCA. Fig. 4 illustrates the handover control procedure when a $\mathrm{MN}$ moves between HCAs' domain.

- The first step for handover control is the network selection process to choose a target PoA for an imminent L2 handover. When the signal strength delivered from PoA1 becomes lower than the predefined threshold value, the MN's MIHF generates an event trigger, called
MIH_Link_LGD, to notify the situation to upper layer protocols.

- On receiving this trigger, the MN invokes a MIH Scan command to search currently reachable PoAs. The search result is delivered from the MN to MICS using a Network Selection Query message.

- The MICS is gathering each AN's network status information (e.g., available resources, channel error rate, link cost, etc.) from the reports periodically offered by PoAs. Based on the information, the MICS prioritizes the reachable PoAs and provides the priority order to the $\mathrm{MN}$ using a Network Selection Response message.

- Then the MN chooses a target PoA for L2 handover (PoA2 in Fig. 2) in consideration of the MICS's recommendation and the pre-defined user preference. A L2 handover can be initiated by the MN with a MIH_HO_Commit command.

- In the handover control procedure, a L2 ID contained in the Location Registration message will be one of the MN's L2 IDs which MICS already obtained through the MN's initial registration procedure. Thus, the MICS can find an existing binding entry relevant to the $\mathrm{MN}$ from its GBT. This enables the MICS to know that the MN is performing a L3 handover.

- Then the MICS records the new CoA of MN (HCA2's IP address) into the relevant binding entry. A Location Registration ACK message delivered from the MICS to HCA2 contains the MN's HoA (which is not NULL) and tunneling information related to the $\mathrm{MN}$ (i.e., CN's HoA and HCA3's IP address).

- Then HCA2 perceives the MN's L3 handover situation and immediately establishes an IP tunnel destined to HCA3 to deliver packets from the MN's new location to the CN.

- While handling the Location Registration message from HCA2, the MICS simultaneously searches its GBT to investigate all the IP tunnels affected by the MN's handover (e.g., a tunnel between HCA1 and HCA3).

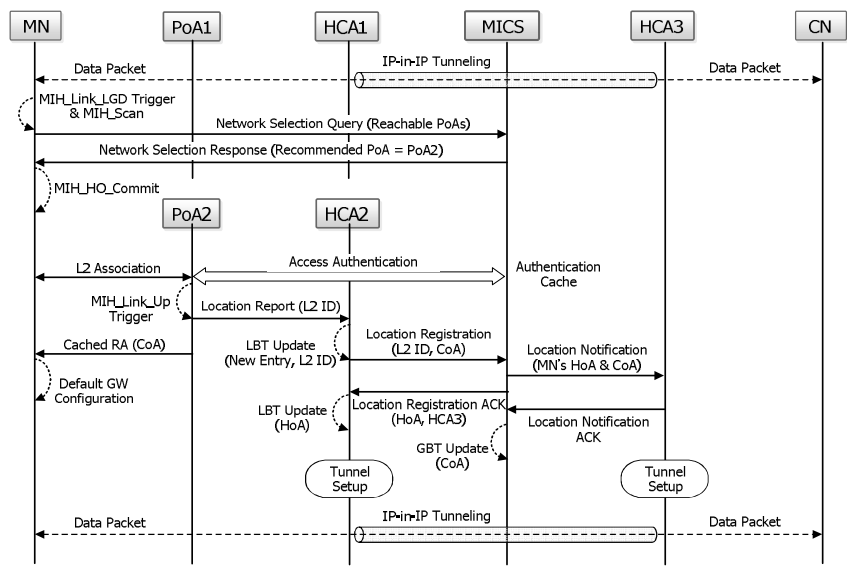

Figure 2. Information Flow in Location Update after Handover

- Then MICS sends a Location Notification message to HCA3 to adjust the relevant IP tunnel. Since this message 
contains the MN's new CoA (HCA2's IP address), HCA3 updates the tunnel's endpoint to be HCA2 instead of HCA1.

- Then HCA3 replies to MICS with a Location Notification ACK message.

- Finally, the MICS completes the handover control procedure by updating the tunnel information in GBT so that an endpoint is changed from HCA1 to HCA2.

\section{Initial Data Transmission procedure}

Fig. 3 depicts the data packet transport procedure between two MNs (i.e., $\mathrm{MN}$ and $\mathrm{CN}$ in the figure) in the AIMS network. It illustrates a situation that a $\mathrm{CN}$ residing in the HCA3's domain transmits packets to a $\mathrm{MN}$ in the HCA1's domain.

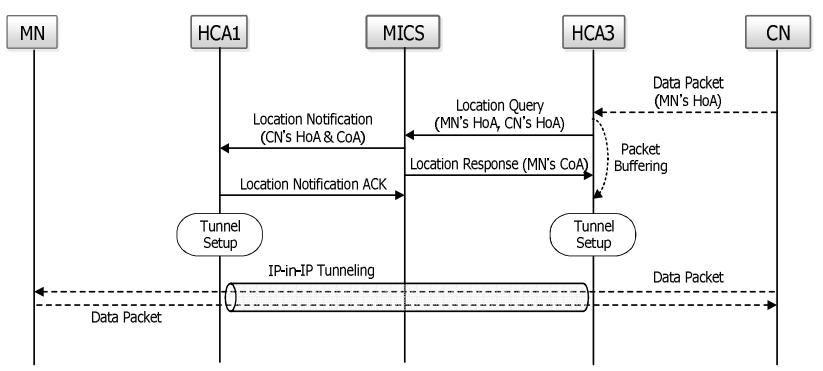

Figure 3. Information Flow in Initial Data Transmission

- We assume the $\mathrm{CN}$ already knows the MN's HoA. To send the packets, the $\mathrm{CN}$ uses the HoA for a destination address of the packets regardless of whether the destination node is a mobile or not.

- These packets are first delivered to HCA3 which is a gateway router of the CN's network. Then HCA3 checks if there exists an IP tunnel coupled with those packets' destination (i.e., MN's HoA). In case that an existing IP tunnel is found, HCA3 immediately encapsulates the packets and sends them to HCA1 through the IP tunnel. Otherwise, HCA3 temporally stores the packets in its buffer and sends a Location Query message to the MICS to know the MN's CoA (i.e., HCA1's IP address).

- When the MICS receives the Location Query message, it searches its own GBT to obtain a CoA bound to the MN's HoA. Then the MICS replies to HCA3 with a Location Response message containing the HCA1's address as the MN's CoA.

- Simultaneously, the MICS sends a Location Notification message to inform HCA1 of the need of a new IP tunnel establishment between HCA1 and HCA3. This notification message contains the CN's HoA and CoA.

- HCA1 replies to MICS with a Location Notification ACK message. Finally, the MICS records the new tunnel information into its GBT to prepare further handovers of the $\mathrm{MN}$ and $\mathrm{CN}$ in the future. This enables MICS to know which HCAs should be notified to adjust their tunnels relevant to a future handover situation.

- With the procedure described above, HCA1 and HCA3 can know each other's IP address to establish a bi-directional IP tunnel between them. The HoAs of $\mathrm{MN}$ and $\mathrm{CN}$ are also exchanged by two HCAs to encapsulate the data packets and forward them into the IP tunnel.

- After establishment of the IP tunnel, HCA3 encapsulates packets being temporally stored in its buffer and forwards them through the IP tunnel.

- Thereafter, HCA1 and HCA3 continuously encapsulate/decapsulate the data packets exchanged by MN and $\mathrm{CN}$. The two HCAs also perform forwarding of the decapsulated packets toward the destination node (i.e., $\mathrm{MN}$ or $\mathrm{CN}$ ) residing within their ANs.

\section{Data Transmission procedure after Handover}

- After a MN's movement, a MN performs L2 association procedure with new PoA to establish a wireless link. Then, a PoA immediately sends to the $\mathrm{MN}$ the cached RA message, which includes address information of HCA, default gateway router in a new IP domain. A MN configures the CoA of HCA as default gateway router and starts sending data to a $\mathrm{CN}$.

- If the location registration procedure between the HCA1 and MICS after a MN's handoff does not complete and the HCA1 receives MN's data packets, the HCA1 temporally stores the packets in its buffer until the location registration procedure completes.

- When the HCA1 receives the location registration ack message from the MICS, the HCA1 performs a new IP tunnel establishment with CNs' HCA. After establishment of the IP tunnel, HCA1 encapsulates packets being temporally stored in its buffer and forwards them through the IP tunnel.

- On the other hand, when a $\mathrm{CN}$ residing in the HCA2's domain transmits packets to a MN in the HCA1, the HCA2 prepares an IP tunnel with the HCA1 through the location notification message. This message, which contains the MN's HoA and CoA, informs HCA2 of the need of a new IP tunnel establishment between HCA1 and HCA2. After establishment of the IP tunnel, HCA2 encapsulates packets and forwards them through the IP tunnel.

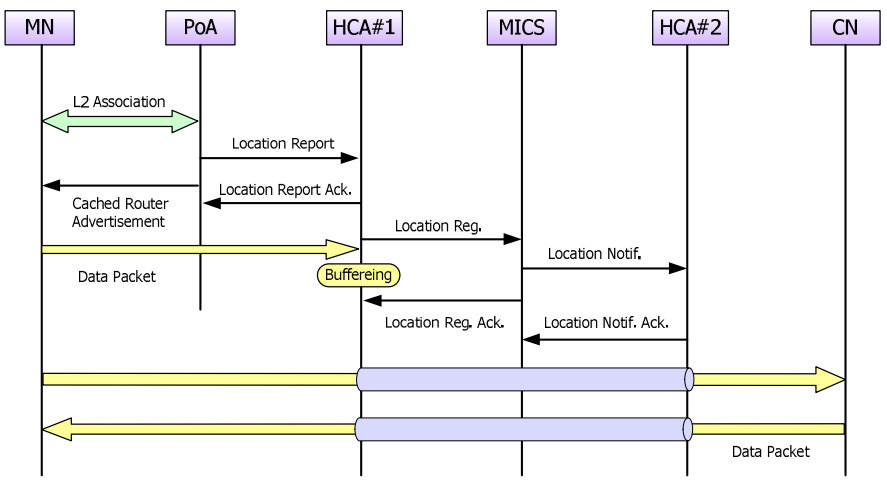

Figure 4. Information Flow in Data Transmission after MN's Handover

\section{III.ENHANCED AIMS}

The AIMS has a structure appropriate to accommodate the VM-based terminal environment on the micro cloud platform. This chapter describes the informational flows of location 
registration and data transmission procedures for the VMbased terminal mobility management.

\section{A. Location registration procedure}

Fig. 5 shows the information flow in location registration. In this procedure, MICS sends a HoA notification message to inform VM-based MN of MN's HoA information. Then, the VM-based MN sends VDI data response after finding out a source address of VDI data request is equal to the HoA.

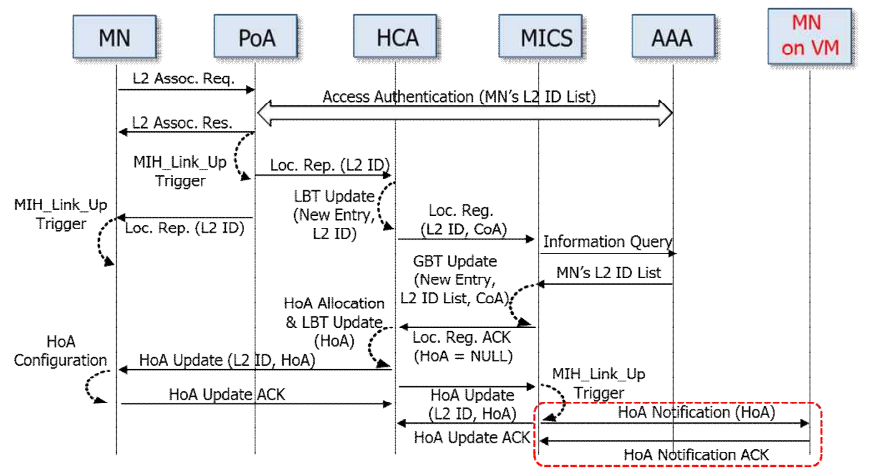

Figure 5. Information flow in location registration

\section{B. Data transmission procedure}

Fig. 6 depicts the information flow in data transmission. In this procedure, $\mathrm{CN}$ sends packets to VM-based $\mathrm{MN}$ instead of $\mathrm{MN}$. The changed data are reflected in screen of VM. When receiving a VDI data request, VM-based $\mathrm{MN}$ sends the updated screen.

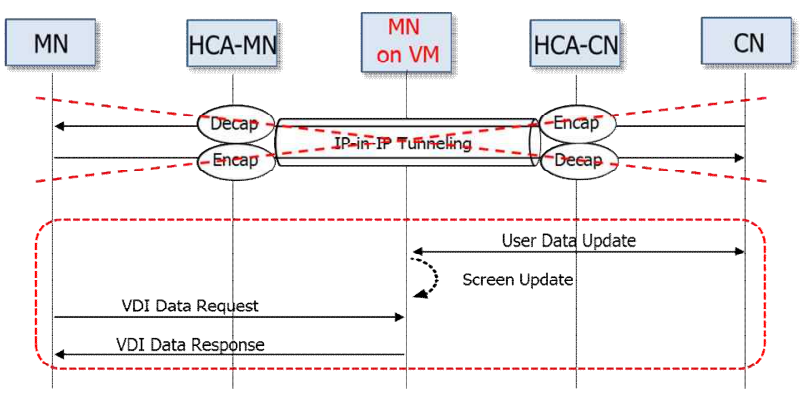

Figure 6. Information flow in data transmission

\section{IV.PERFORMANCE IMPROVEMENT}

By enhancing the procedures for the AIMS, we can eliminate the data loss as well as retain the application sessions during the handover. Also, we do not need to establish the data tunneling for the data transmission between a mobile node and a network node.

\section{CONCLUSION}

In this paper, we propose the enhancement to apply the VM-based terminal mobility management to the AIMS in the procedure for location management and handover management. In detail, the information flow in the procedure for location management and handover management newly introduced location notification message to VM-based mobile terminal and removed unnecessary data tunneling between routers.

\section{ACKNOWLEDGEMENT}

This research was funded by the MSIP(Ministry of Science, ICT \& Future Planning), Korea in the ICT R\&D Program 2013

\section{REFERENCES}

[1] C. Perkins, "IP Mobility Support for IPv4," RFC3344 on IETF, Aug 2002

[2] Myoungju Yu et al., "A New Mechanism for Seamless Mobility Based on MPLS LSP in BcN," IEICE Transactions on Communications, Vol. E91-B, No. 2, Feb. 2008.

[3] S. Gundavelli et al., "Proxy Mobile IPv6," RFC5213 on IETF, Aug 2008

[4] R. Wakikawa and S. Gundavelli, "IPv4 Support for Proxy Mobile IPv6," Internet Draft on IETF, Sept. 2009

[5] V. Devarapalli et al., "Multiple Interface Support with Proxy Mobile IPv6," Internet Draft on IETF, Sept. 2009.

[6] K. Hong et al., "A study on distributed mobility management to improve centralized location management problem," ICTC 2011. 